\title{
Atuação fonoaudiológica durante a radioterapia em pacientes com câncer de cabeça e pescoço
}

\author{
Caroline P. dos Santos ${ }^{1^{\star}}$
}

\begin{abstract}
Resumo
O presente trabalho tem o objetivo de refletir e discutir através de dados científicos sobre os limites e benefícios da atuação fonoaudiológica durante a radioterapia em pacientes com câncer de cabeça e pescoço. A radioterapia na região da cabeça e pescoço pode desencadear diferentes graus de severidade de sequelas, os quais podem permanecer por até dois anos quando não estimulados. A atuação fonoaudiológica busca minimizar o impacto causado pelo tratamento oncológico e engloba orientações, encaminhamentos, monitoramentos e terapia, objetivando proporcionar uma melhora na qualidade de vida. Para isto, o fonoaudiólogo faz uso de estratégias e adaptações visando uma maior interação com os familiares, através da comunicação, e o prazer de uma alimentação por via oral de maneira segura.
\end{abstract}

Descritores: Neoplasias de cabeça e pescoço; Radioterapia; Fonoaudiologia; Transtornos de deglutição; Disfonia.

\section{Abstract \\ Speech therapy during radiotherapy in patients with head and neck cancer}

This paper aims to reflect upon and discuss scientific data on the limits and benefits of speech therapy during radiotherapy in patients with head and neck cancer. Radiation therapy in the head and neck can trigger sequelae of different degrees of severity, which may persist for up to two years in the absence of stimulation. The speech therapy seeks to minimize the impact caused by cancer treatment and includes guidance, referrals, monitoring, and therapy focused on provide a better quality of life. For this purpose the speech therapist makes use of strategies and adaptations aimed at greater interaction with the family through communication and the pleasure of a safe oral feeding.

Keywords: Head and neck neoplasms; Radiotherapy; Speech, language and hearing sciences; Deglutition disorders; Dysphonia.
1. Serviço de Fonoaudiologia. Hospital Universitário Pedro Ernesto. Universidade do Estado do Rio de Janeiro. Rio de Janeiro, RJ, Brasil.

*Endereço para correspondência:

Avenida Embaixador Abelardo Bueno, 2.510, 2/810

Rio de Janeiro, RJ, Brasil. CEP: 22775-040.

E-mail: carolinepeixoto.27@gmail.com

Revista HUPE, Rio de Janeiro, 2015;14(Supl. 1):73-79

doi:10.12957/rhupe.2015.17930

Recebido em 03/06/2015. Aprovado em 15/07/2015.

\section{Resumen}

Actuación fonoaudiológica durante la radioterapia en pacientes con cáncer de cabeza y cuello

El presente trabajo tiene el objetivo de reflexionar y discutir, a través de datos científicos, sobre los límites y beneficios de la actuación fonoaudiológica durante la radioterapia en pacientes con cáncer de cabeza y cuello. La radioterapia en la región de la cabeza y el cuello puede desencadenar diferentes grados de severidad en las secuelas, los cuales pueden permanecer durante 2 años cuando no son estimuladosLa actuación fonoaudiológica busca minimizar el impacto causado por el tratamiento oncológico e incluye orientaciones, derivaciones, monitoreo y terapia con el objetivo de proporcionar una mejora en la calidad de vida. Para esto, el fonoaudiólogo hace uso de estrategias y adaptaciones destinadas a una mayor interacción con los familiares a través de la comunicación y el placer de una alimentación por vía oral de forma segura.

Palabras clave: Neoplasias de cabeza y cuello; Radioterapia; Fonoaudiología; Trastornos de deglución; Disfonía. 


\section{Artigo de revisão}

\section{Introdução}

A participação da fonoaudiologia em equipe multidisciplinar no Centro Universitário de Controle do Câncer (CUCC) do Hospital Universitário Pedro Ernesto (HUPE) se deu em 2007 pela necessidade de atender às dificuldades de voz, fala e, principalmente, de deglutição em pacientes oncológicos em região de cabeça e pescoço submetidos à radioterapia. Ao longo dos anos, o trabalho da fonoaudiologia nesses pacientes foi se ampliando e desta forma ocorreu a inserção em outras equipes, como no Ambulatório de Cirurgia de Cabeça e Pescoço e no Núcleo de Cuidados Paliativos (NCP). A atuação fonoaudiológica em ambulatório de cirurgia de cabeça e pescoço visa a triagem e acompanhamento nas fases pré-cirúrgica ou pré-radioterápica, com avaliações estruturais e funcionais da deglutição, orientações sobre a reabilitação proposta após procedimento cirúrgico e adaptações na deglutição quando necessário. No NCP, atualmente, a fonoaudiologia está inserida por meio de pareceres e encaminhamentos para acompanhamento ambulatorial, contribuindo na readaptação para uma alimentação segura e desenvolvendo métodos ou estratégias para melhor comunicação do paciente. ${ }^{1}$

A reabilitação oncológica se faz necessária também junto aos pacientes que foram submetidos à radiação, pois são descritas sequelas presentes após estes tratamentos que envolvem dificuldades na comunicação oral e/ou deglutição. ${ }^{2}$

A radioterapia na região da cabeça e pescoço pode acarretar diferentes disfunções em diferentes graus de severidade, como edema e fibrose da região exposta, trismo, mucosite, xerostomia, odinofagia, dermatite actínica, perda ponderal e a necessidade do uso de vias alternativas de alimentação. ${ }^{3}$ Alterações de voz e de deglutição podem ser justificadas pelas mudanças na sensibilidade e mobilidade das estruturas afetadas pela radiação decorrente do edema, fibrose e redução da elevação laríngea. ${ }^{46}$ Desta forma, estes distúrbios, independentemente do grau de severidade, podem desencadear modificações em diferentes aspectos da vida do indivíduo e interferir na sua qualidade., ${ }^{3,-10}$ Sendo assim, cabe ao fonoaudiólogo maximizar e adaptar a deglutição, preservando com segurança o prazer da alimentação por via oral. Quanto à comunicação, vale, por vezes, adaptá-la visando uma maior integração social e familiar.

\section{Metodologia}

Diante do exposto, realizou-se pesquisa bibliográfica em bases de dados científicos eletrônicos, objeti- vando expor e discutir através de dados de diversas pesquisas sobre as reações agudas ao tratamento radioterápico e os limites e benefícios do gerenciamento fonoaudiológico durante este processo em pacientes com câncer de cabeça e pescoço.

\section{Reações à radioterapia e repercussões fonoaudiológicas}

A radioterapia na região da cabeça e pescoço pode desencadear diferentes graus de severidade de sequelas agudas, como mucosite, candidíase, xerostomia, ulcerações e sangramento da mucosa, que explicam as dificuldades na mastigação, no controle sensorial e motor do alimento e na redução da amplitude do movimento e força da língua, faringe e laringe. Em relação às sequelas tardias, as mais referidas são o edema da laringe, fibrose, rigidez, radionecrose, trismo, cáries dentárias, xerostomia, redução do olfato e do paladar, ${ }^{11-13}$ paralisia de prega vocal, diminuição do reflexo de deglutição e do peristaltismo faríngeo, os quais podem permanecer por até dois anos quando não estimulados. ${ }^{13}$ Todas estas alterações podem promover modificação tanto da eficiência quanto da segurança da deglutição, resultando nas disfagias. 11,12

O edema, a fibrose, ou a irregularidade das bordas da mucosa da prega vocal podem resultar em disfonia, ${ }^{10,11}$ em que as principais queixas da comunicação oral são fadiga, redução da extensão do pitch e da loudness, falta de clareza vocal, rouquidão e desvantagem vocal nos aspectos social, funcional, emocional e físico. ${ }^{11} \mathrm{Em}$ estudo prospectivo, $80 \%$ dos pacientes que foram avaliados apresentaram alteração na qualidade vocal após um ano do tratamento de radioterapia. ${ }^{14}$

A mucosite, inflamação da mucosa da cavidade oral, é o efeito agudo de maior frequência, que, além de dolorosa, interfere na alimentação adequada do paciente, podendo haver necessidade de nutrição parenteral e analgesia. ${ }^{15,16}$ Como sintoma imediato da ação radioterápica, pode ocorrer sensação dolorosa durante a deglutição, tanto pela irritação dos tecidos como pelas dificuldades presentes nas tentativas de elevação do complexo laríngeo. Na fonação, destaca-se dificuldade vibratória decorrente das mucosites. ${ }^{15}$

A xerostomia, a fibrose, a redução do movimento e da força muscular e as alterações sensoriais, reações à radioterapia, são as mais citadas como causas da disfagia e justificam as anormalidades das fases preparatória, oral e faríngea da deglutição, incluindo a presença de penetração e/ou aspiração, durante e após o tratamento., 5,12,17,18-20 A longo prazo, a fibrose pode dificultar o movimento 
de elevação da laringe no pescoço, comprometendo o desempenho da função de proteção da via aérea inferior no momento da deglutição e da modulação vocal, quanto à função fonatória. ${ }^{21}$

Quanto à análise funcional, realizada em pacientes com câncer de orofaringe submetidos a tratamento curativo (radioterapia, quimioterapia e cirurgia, exclusivas ou associadas entre si, com ou sem reconstrução), observaram-se sistema estomatognático com alterações de sensibilidade e mobilidade em língua, lábios, palato mole, regiões jugais e também da simetria de face, lábios e língua; discreta alteração da qualidade vocal, porém com presença de alteração de inteligibilidade da fala e de erros articulatórios, como de golpe de glote e fricativas faríngeas; e deglutição com alteração da fase oral e faríngea, caracterizando uma disfagia discreta. ${ }^{22}$

As alterações da deglutição observadas nos pacientes com câncer de cabeça e pescoço submetidos à videofluoroscopia são caracterizadas por imobilidade da epiglote, alteração do movimento da faringe, da elevação da laringe, do movimento de retração da base da língua, modificação do tempo de duração da deglutição, incluindo aumento do tempo da fase oral para a preparação do bolo, aumento do tempo do trânsito faríngeo, atraso da elevação do osso hioide e alterações no clareamento faríngeo, resultando nas estases em valécula e nos seios piriformes, apresentando penetração laríngea e/ou aspiração traqueal em alguns casos. ${ }^{12,17-19,23-25}$ Estas alterações, por sua vez, podem resultar em disfagia e consequente desnutrição, desidratação, pneumonia aspirativa e restringir a qualidade de vida do indivíduo devido à limitação de diversos aspectos, como emocional, social, ocupacional, físico e funcional. ${ }^{26}$

Vale ressaltar a importância de uma equipe multidisciplinar composta de cirurgiões, radioterapeutas, oncologistas clínicos, enfermeiros, nutricionistas, fonoaudiólogos, dentistas, fisioterapeutas e psicólogos no tratamento dos carcinomas da cabeça e do pescoço, objetivando atingir mais efetivamente a reabilitação dos deficit funcionais decorrentes do tratamento ${ }^{20,27} \mathrm{e}$, dessa forma, minimizar os impactos na qualidade de vida desses pacientes.

\section{Reabilitação fonoaudiológica - Limites e benefícios da atuação fonoaudiológica}

Numa abordagem isolada das sequelas da radioterapia, torna-se importante estimular a ação vibratória da mucosa de pregas vocais, bem como tentativas de mobilização da laringe (elevação), por estratégias de emissões melódicas ou manobras de deglutição. ${ }^{21}$

\section{Voz e fala}

Um planejamento terapêutico fonoaudiológico para a manutenção e reabilitação da comunicação oral durante a radioterapia foi descrito em um estudo que teve como foco a qualidade vocal e orientações quanto à higiene vocal, redução do esforço e da fadiga vocal. Os autores sugeriram métodos de relaxamento e mobilidade dos músculos do trato vocal e da laringe, e exercícios de vibração para a mucosa de revestimento das pregas vocais. ${ }^{11}$

Durante o tratamento irradiante, o paciente passa por um período de extremo desconforto, com muita dor às tentativas de deglutição e fonação, de forma que as sessões de fonoterapia podem ser interrompidas para evitar o agravamento de tal situação. Nesse momento, também apresenta dificuldades em atingir resultados satisfatórios na execução das estratégicas propostas. ${ }^{21}$

Um estudo randomizado analisou pacientes com câncer glótico inicial tratados com radioterapia exclusiva ou cirurgia a laser com 23 indivíduos, sendo 12 submetidos à terapia vocal e 11 do grupo sem terapia. A terapia foi baseada em orientações quanto à higiene vocal, exercícios para respiração e vocais propriamente ditos. Os autores concluíram que a terapia vocal beneficia os pacientes com disfonia após o tratamento para carcinoma glótico inicial. Porém, a maior parte das variáveis do estudo não apresentou diferença significativa entre os grupos. ${ }^{28}$

Vários estudos, nos últimos anos, descreveram as alterações vocais decorrentes da radioterapia e comentaram a necessidade da intervenção fonoaudiológica durante e/ou após a radioterapia, objetivando minimizar as sequelas do tratamento. ${ }^{11,28,30}$ Porém, ainda não está claro o quanto a terapia vocal pode beneficiar os pacientes com disfonia após a radioterapia, nem mesmo o momento indicado de iniciá-la. ${ }^{3}$

A fonoterapia durante a radioterapia ou num póstérmino imediato poderia ser também melhor compreendida, associada à análise dos exames laringológicos, que foi a intenção inicial, inviabilizada devido às dificuldades técnicas encontradas essencialmente pela presença de edema. Talvez edemas discretos possam responder à terapia vocal, mas edemas graves, no mínimo, não respondem à reabilitação, como observado clinicamente com pacientes submetidos a tratamentos mais agressivos de radioterapia e quimioterapia que não respondem ao tratamento medicamentoso para edemas 


\section{Artigo de revisão}

graves. A observação clínica, entretanto, sugere que a fonoterapia apresenta respostas positivas quando realizada num tempo maior de pós-término do tratamento. ${ }^{3}$

\section{Deglutição}

A alimentação tem importância nutricional e social. As disfagias associadas ao tratamento radioterápico ou cirúrgico nas regiões de cavidade oral, orofaringe e laringe, quando prontamente identificadas e avaliadas, permitem uma reabilitação mais rápida e efetiva da deglutição via oral, além da possibilidade de restabelecimento do estado nutricional do paciente e sua possível reintegração social. ${ }^{20}$

Em um estudo observaram-se resultados satisfatórios quanto à realização de terapia para deglutição antes, durante e após a radioterapia em pacientes de câncer de cabeça e pescoço, em qualquer estágio, cuja proposta de terapia para deglutição era composta por orientações pré-radioterapia sobre a fisiologia da deglutição e as sequelas agudas e tardias que poderiam se manifestar em diferentes graus de severidade, comprometendo a função. Os pacientes foram orientados quanto à importância da realização dos exercícios e a utilização das posturas e manobras de proteção durante a alimentação no período do tratamento e até um mês após seu término. Como terapia indireta, foi indicada estimulação sensorial intraoral (tátil e térmica), exercícios para a mobilidade anteroposterior da língua, mobilidade dos bucinadores, força de base de língua e do bucinador, elevação da laringe e contração da faringe. Como terapia direta, foi orientada adaptação de consistência, temperatura, quantidade e viscosidade, conforme disfunção individual: uso de posturas facilitadoras para o transporte do bolo (cabeça inclinada ou virada); manobras de proteção das vias aéreas inferiores (esforço, super-supraglótica e Mendelsohn); e manobras de limpeza (deglutições múltiplas e alternância com líquido). ${ }^{3}$

São descritas na literatura o uso de posturas e manobras de proteção, como esforço, super-supraglótica, Mendelsohn e Masako, associadas ou não à mudança de consistência e viscosidade, que contribuem para a melhora da deglutição. ${ }^{11,31,32}$ A efetividade da manobra de mudança de postura foi descrita por Lazarus e colaboradores, ocasião na qual eliminou-se a aspiração de cinco dos oito pacientes tratados com radioterapia e quimioterapia adjuvante. ${ }^{12}$ Para Eisbruch e colaboradores, apenas os exercícios e as manobras de proteção já são mecanismos vantajosos para minimizar o risco das aspirações e da pneumonia aspirativa, resultando em uma deglutição segura. ${ }^{20}$

Em estudo com casuística reduzida foram avaliados, por meio do exame de videofluoroscopia, nove pacientes, cuja observação permitiu concluir que a manobra de proteção super-supraglótica melhora o padrão da deglutição de pacientes irradiados na região de cabeça e pescoço. ${ }^{31}$

Em um outro estudo foi descrito que manobras de postura, de proteção das vias aéreas e de limpeza podem ser orientadas desde o pré-tratamento. No póstratamento é indicada avaliação clínica e videofluoroscópica da deglutição com o objetivo de detalhar as causas, caracterizar e quantificar as alterações para uma abordagem objetiva nos deficit diagnosticados. ${ }^{11}$

Exercícios para a musculatura oral são indicados para melhorar a integração sensório-motora, a força e a dinâmica do movimento da musculatura afetada. ${ }^{31,32}$ Técnicas para abertura de boca foram relatadas para evitar o trismo, desde o pré-tratamento. ${ }^{11}$

Os autores sugeriram como profilaxia, objetivando maximizar a função da deglutição, exercícios para melhorar a retração da base da língua, o aumento do contato da base da língua com a parede posterior da faringe, a elevação laríngea e manobras de proteção, como esforço, super-supraglótica e Mendelsohn. ${ }^{24}$

Neste estudo, 37 pacientes foram acompanhados no período pré e pós-radioterapia, dos quais 25 foram submetidos à terapia para deglutição duas vezes por semana por 14 meses desde o pré-tratamento e 12 pacientes fizeram terapia após o término da radioterapia. Os resultados demonstraram melhores efeitos no grupo que fez terapia pré-radioterapia, porém os próprios autores solicitaram cautela na interpretação dos resultados por não se tratar de estudo randomizado e devido à ausência de padronização no momento de aplicação do questionário pós-terapia. ${ }^{33}$

A terapia fonoaudiológica para a deglutição de pacientes submetidos ao tratamento com radioterapia associada à quimioterapia vem evoluindo e apresentando maior efetividade, $11,12,20,24,27,32,34$ porém a maior parte dos estudos carece de análises objetivas de eficácia. ${ }^{3}$

A reabilitação da deglutição realizada antes, durante e após a radioterapia em pacientes com câncer de cabeça e pescoço pode contribuir para melhorar a fase preparatória e oral e a qualidade de vida de pacientes tratados por câncer de cabeça e pescoço. ${ }^{3}$

Qualidade de vida

A importância da qualidade de vida dos pacientes pós-tratamento de câncer de cabeça e pescoço tem sido 
um assunto pesquisado por fonoaudiólogos, visto a forte ligação da comunicação e a integração social. Esta última, às vezes, ausente nestes pacientes em virtude da desfiguração facial. Pesquisas trazem resultados importantes de alteração da qualidade de vida de pacientes submetidos a tratamentos diversos em cabeça e pescoço quando comparados com a população normal. ${ }^{2}$

A avaliação da qualidade de vida em pacientes com câncer de cabeça e pescoço geralmente é realizada pela aplicação de questionários específicos, sendo considerado um método eficaz de avaliação dos resultados terapêuticos referentes aos aspectos físicos, funcionais e psicossociais. ${ }^{35}$ Questionários de qualidade de vida foram aplicados a pacientes tratados com câncer de orofaringe que apresentaram boa qualidade de vida relacionada à voz, avaliados por ambos os questionários: Voice Handicap Index (VHI) e da versão em português (QVV) do Voice-Related Quality of Life(V-RQOL), além do impacto negativo da qualidade de vida relacionado à deglutição avaliado através do questionário Quality of Life in Swallowing Disorders (SWAL-QOL). ${ }^{22}$

A percepção do paciente em relação às dificuldades na deglutição está associada às alterações visualizadas na videofluoroscopia e, portanto, esta pode ser utilizada como ferramenta do status da disfagia no decorrer do tratamento ${ }^{23}$ Clinicamente, as escalas funcionais de disfagia vêm sendo utilizadas para acompanhar a evolução da disfagia. Por exemplo, a escala funcional de O'Neil, cuja classificação está subdividida em sete níveis desde disfagia severa a deglutição sem alterações. ${ }^{36}$

A necessidade do serviço de fonoaudiologia é reforçada ao verificar estudo que mensurou através de uma escala de ansiedade e depressão aplicada a cônjuges e pacientes tratados para câncer de cabeça e pescoço. Seus resultados indicaram um percentual de 20\% dos parceiros e de $27 \%$ dos pacientes com respostas positivas à escala de ansiedade e depressão. A porcentagem de respostas desfavoráveis dos pacientes foi relacionada à presença da sonda para alimentação, fala não bem expressiva, contatos sociais menores e um estilo passivo de lidar com atividades diárias e com as emoções. Este mesmo padrão de respostas entre os cônjuges foi relacionadoà presença da sonda nasogástrica para alimentação de seus parceiros, um estilo de vitalidade menor e mais passivo e pouca interação às atividades da vida diária. ${ }^{37}$

A atuação fonoaudiológica é de suma importância, visto que tanto o tumor de cabeça e pescoço quanto o tratamento afetam aspectos da vida diária e resultam na piora da qualidade de vida. $\mathrm{O}$ acompanhamento fonoaudiológico na fase pré ou pós-radioterapia visa a preservação ou readaptação das funções comunicativas do indivíduo, o que traz claro impacto na qualidade de vida destes. ${ }^{2}$

\section{Conclusão}

O gerenciamento fonoaudiológico durante o tratamento radioterápico compreende orientações, monitoramentos, encaminhamentos, fonoterapia em alguns casos e educação continuada. Dentre as possíveis reações agudas e tardias apresentadas pelos pacientes com história de câncer de cabeça e pescoço submetidos à radioterapia, o gerenciamento fonoaudiológico atua, principalmente, nas áreas da voz e deglutição. Sendo assim, o fonoaudiólogo deve direcionar esforços que visem contribuir no processo de recuperação, minimizando os danos causados pela doença e melhorando a condição de saúde do indivíduo.

Quanto à voz, podemos observar divergências em relação à realização de fonoterapia durante processo radioterápico. Neste aspecto, o gerenciamento fonoaudiológico compreende, principalmente, orientações quanto às reações agudas, como disfonias, higiene vocal e hidratação. Em suma, minimizar as sequelas presentes que interferem na comunicação oral.

Quanto à deglutição, compreende o uso de manobras compensatórias, estimulações sensório-motoras orais, encaminhamentos para enfermagem, bucomaxilofacial e nutricionistas, mudanças de consistência alimentar, fracionamento das dietas, sugestão de via alternativa para alimentação, sendo condutas adotadas durante o processo de radioterapia que contribuam para manter a qualidade de vida e social destes pacientes.

A aplicação de protocolos com relação à voz e deglutição mostra-se uma estratégia a mais a ser utilizada para contribuir na manutenção da qualidade de vida de pacientes com câncer de cabeça e pescoço.

De acordo com as reações agudas apresentadas durante radioterapia pelos pacientes com câncer de cabeça e pescoço, a atuação fonoaudiológica pode estar limitada, principalmente, quanto à fonoterapia vocal. Entretanto esta atuação no monitoramento da deglutição vem apresentando benefícios para manutenção da qualidade de vida destes pacientes.

São necessários novos estudos que comparem qualidade de vida, gerenciamento fonoaudiológico, voz e deglutição dos pacientes submetidos ao tratamento fonoaudiológico e grupos-controles, bem como protocolos de avaliação, encaminhamentos e intervenção dos casos provenientes do ambulatório de radioterapia. 


\section{Artigo de revisão}

\section{Agradecimento}

À fonoaudióloga Catiuscia Greco.

\section{Referências}

1. Calheiros AS, Albuquerque CL. A vivência da fonoaudiologia na equipe de cuidados paliativos de um hospital universitário do Rio de Janeiro. RHUPE. 2012 abr-jun;11:94-98.

2. Campos RJDS, Leite ICG. Qualidade de vida e voz pós-radioterapia: repercussões para a fonoaudiologia. Revista CEFAC. 2010 jul-ago;12(4):671-677.

3. Barros AP. Efetividade da reabilitação fonoaudiológica na voz e na deglutição em pacientes irradiados devido ao câncer de cabeça e pescoço (tese de doutorado). São Paulo: Fundação Antônio Prudente; 2007.

4. Pauloski BR, Rademaker AW, Logemann JA, et al. Speech and swallowing in irradiated and nonirradiated postsurgical oral cancer patients. Otolaryngol Head Neck Surg. 1998;118:616-24.

5. Logemann JA, Pauloski BR, Rademarker AW, et al. Xerostomia: 12-month changes in saliva production and its relationship to perception and performance of swallow function, oral intake, and diet after chemoradiation. Head Neck. 2003;25:432-7.

6. Logemann JA, Smith CH, Pauloski BR, et al. Effects of xerostomia on perception and performance of swallow function. Head Neck. 2001;23:317-21.

7. Tschudi D, Stoeckli S, Schmid S. Quality of life after different treatment modalities for carcinoma of the oropharynx. Laryngoscope. 2003;113:1949-54.

8. Meyer TK, Kuhn JC, Campbell BH, et al. Speech intelligibility and quality of life in head and neck cancer survivors. Laryngoscope. 2004;114:1977-81.

9. Gillespie MB, Brodsky MB, Day TA, et al. Laryngeal penetration and aspiration during swallowing after the treatment of advanced oropharyngeal cancer. Arch Otolaryngol Head Neck Surg. 2005;131:615-9.

10. Murphy BA, Ridner S, Wells N, et al. Quality of life research in head and neck cancer: a review of the current state of the science. Crit Rev Oncol Hematol. 2007;62:251-67.

11. Samlan RA, Webster KT. Swallowing and speech therapy after definitive treatment for laryngeal cancer. Otolaryngol Clin North Am. 2002;35:1115-33.

12. Lazarus CL, Logeman JA, Pauloski BR, et al. Swallowing disorders in head and neck cancer patients treated with radiotherapy and adjuvant chemo therapy. Laryngoscope. 1996;106(9):1157-66.

13. Carrara-De-Angelis E, Mourão LF, Furia CLB. Disfagias associadas ao tratamento do câncer de cabeça e pescoço. Acta Oncológica Brasileira. 1997;17(2):77-82.

14. Caminero CMJ, Señaris GB, López LA, et al. Voice quality assessment after larýngeal câncer radiotherapeutic treatment at initial stages. Clin Transl Oncol. 2006;8(4):284-9.

15. Meyer TK, Kuhn JC, Campbell BH, et al. Speech intelligibility and quality of life in head and neck cancer survivors. Laryngoscope. 2004;114:1977-81.

16. Labbate R, Lehn C, Denardin O. Efeito da clorexidina na mucosite induzida por radioterapia em câncer de cabeça e pescoço. Rev Bras Otorrinolaringol. 2003:69(3):349-54.

17. Kotz T, Abraham S, Beitler JJ, et al. Pharyngeal transport dysfunction consequent to an organ-sparing protocol. Arch Otolaryngol Head Neck Surg. 1999;125:410-3.
18. Smith RV, Kotz T, Beitler JJ, et al. Long-term swallowing problems after organ preservation therapy with concomitant radiation therapy and intravenous hydroxyurea. Arch Otolaryngol Head Neck Surg. 2000;126:384-9.

19. Newman LA, Robbins KT, Logemann JA, et al. Swallowing and speech ability after treatment for head and neck cancer targeted intraarterial versus intravenous chemoradiation. Head Neck. 2002;24:68-77.

20. Eisbruch A, Schwartz M, Rasch C, et al. Dysphagia and aspiration after chemoradiation for head and neck cancer: which anatomic structures are affected and can they be spared be spared by IMTR?. Int J Radiat Oncol Biol Phys. 2004;60:1425-39.

21. Camargo Z. Reabilitação fonoaudiológica em câncer de laringe. In: Pinho S. Fundamentos de fonoaudiologia - Tratando os distúrbios da voz. $2^{\mathrm{a}}$ ed. Rio de Janeiro: Guanabara Koogan S.A. 2003. p.101-105.

22. Bandeira AKC. Análise funcional e qualidade de vida relacionada à voz e à deglutição de pacientes tratados por câncer de orofaringe (tese de doutorado). São Paulo: Fundação Antônio Prudente; 2007.

23. Pauloski BR, Rademaker AW, Logemann JL, et al. Swallow function and perception of dysphagia in patients with head and neck cancer. Head Neck. 2002;24:555-65.

24. Kotz T, Costello R, Li Y, et al. Swallowing dysfunction after chemoradiation for adavanced squamous cell carcinoma of the head and neck. Head Neck. 2004;26:365-72.

25. Graner DE, Foote RL, Kasperbauer JL, et al. Swallow function in patients before and after intra-arterial chemoradiation. Laryngoscope. 2003;113:573-9.

26. Ding R, Logemann JA. Pneumonia in stroke patients: a retrospective study. Dysphagia. 2000;15:51-7.

27. Lee H, Havrila C, Bravo V, et al. Effect of oral nutritional supplementation on weight loss and percutaneous endoscopic gastrostomy tube rates in patients treated with radiotherapy for oropharyngeal carcinoma. Support Care Cancer. 2008;16:285-289.

28. Van Gogh CD, Verdonck-de Leeuw IM, Boon-Kamma BA, et al. The efficacy of voice therapy in patients after treatment for early glottic carcinoma. Cancer. 2006;106:95-105.

29. Estrela F, Elias V, Martins V. Reabilitação do paciente disfágico em cirurgia de cabeça e pescoço. In: Jacobi J, Levy D, Silva L Disfagia: Avaliação e tratamento. Rio de Janeiro: Revinter.1996; 233-276.

30. Fex S, Henriksson B. Phoniatric treatment combined with radiotherapy of laryngeal cancer for the avoidance of radiation damage. Acta Otolaryngol Suppl. 1969;263:128-9.

31. Logemann JA, Pauloski BR, Rademaker AW, et al. Super-supraglottic swallow in irradiated head and neck cancer patients. Head e Neck J. 1997;19(6):535-40.

32. Mittal BB, Pauloski BR, Haraf DJ, et al. Swallowing dysfunction-preventative and rehabilitation strategies in patients with head and neck cancer treated with surgery, radiotherapy, and chemotherapy: a critical review. Int J Radiat Oncol Biol Phys. 2003;57:1219-30.

33. Kulbersh BD, Rosenthal EL, NcGrew BM, et al. Pretreatment, preoperative swallowing exercises may improve dysphagia quality of life. Laryngoscope. 2006;116:883-6.

34. Nguyen NP, Moltz CC, Frank C, et al. Dysphagia following chemoradiation for locally advanced head and neck cancer. Ann Oncol. 2004;15:383-8.

35. Vartanian J, Carvalho A, Furia C, et al. Questionários para a avaliação de qualidade de vida em pacientes com câncer de cabeça e pescoço validados no Brasil. Rev. Bras. Cir. Cabeça 
Caroline P. dos Santos. • Atuação fonoaudiológica durante a radioterapia em pacientes com câncer de cabeça e pescoço

Pescoço. 2007;36(2):108-115.

36. O'Neil KH, Purdy M, Falk J, et al. The dysphagia outcome and severity scale. Dysphagia 1999;14:139-45.

37. Verdonck-De Leeuw IM, Eerenstein SE, Van Der Linden MH, et al. Distress in spouswes and patients after treatment for head and neck câncer. Laryngoscope. 2007;117(2):238-41 